PROCEEDINGS OF THE

AMERICAN MATHEMATICAL SOCIETY

Volume 133, Number 2, Pages 425-429

S 0002-9939(04)07744-5

Article electronically published on September 2, 2004

\title{
ON SUBCLASSES OF WEAK ASPLUND SPACES
}

\author{
ONDŘEJ F. K. KALENDA AND KENNETH KUNEN
}

(Communicated by Jonathan M. Borwein)

\begin{abstract}
Assuming the consistency of the existence of a measurable cardinal, it is consistent to have two Banach spaces, $X, Y$, where $X$ is a weak Asplund space such that $X^{*}$ (in the weak* topology) in not in Stegall's class, whereas $Y^{*}$ is in Stegall's class but is not weak* fragmentable.
\end{abstract}

A Banach space $X$ is weak Asplund if every convex continuous real function on $X$ is Gâteaux differentiable at all points of a dense $G_{\delta}$ set. This is a large class of spaces; it contains for example all Asplund spaces and all weakly compactly generated spaces. A detailed study of weak Asplund spaces and their subclasses is given in [1. The largest known subclass of Asplund spaces with reasonable stability properties was introduced by C. Stegall [16].

A topological space $T$ belongs to Stegall's class if for any Baire space $B$ and any upper-semicontinuous nonempty compact-valued mapping $\varphi: B \rightarrow T$ which is minimal with respect to inclusion, $\varphi(b)$ is a singleton for all $b \in B$ except for a meager set. If $\left(X^{*}, w^{*}\right)$ is in Stegall's class, then $X$ is weak Asplund. The converse does not hold by [7].

A further subclass is the class of those $X$ such that $\left(X^{*}, w^{*}\right)$ is fragmentable. Recall that a topological space $T$ is fragmentable if there exists a metric $\rho$ on the set $T$ such that every nonempty subset of $T$ has nonempty relatively open subsets of arbitrarily small $\rho$-diameter. Every fragmentable topological space is easily seen to belong to Stegall's class. An example of a Banach space $X$ such that $\left(X^{*}, w^{*}\right)$ is in Stegall's class but not fragmentable is given in [9].

Both results of [9] and [7] use some additional axioms beyond ZFC. As remarked in [7, these two sets of axioms cannot hold at the same time. In the present paper, we construct a model of ZFC in which all three classes - weak Asplund spaces, spaces with dual in Stegall's class, and spaces with weak* fragmentable dual - are mutually different. The main result is contained in the following theorem.

Theorem. If " $Z F C+$ there is a measurable cardinal" is consistent, then "ZFC+ there are Banach spaces $X$ and $Y$ such that $X$ is weak Asplund but $\left(X^{*}, w^{*}\right)$ is

Received by the editors October 4, 2001

2000 Mathematics Subject Classification. Primary 46B26, 03 E35.

Key words and phrases. Weak Asplund space, fragmentable space, Stegall's class of spaces, measurable cardinal.

The first author was supported by Research grants GAUK 277/2001, GAČR 201/00/1466 and MSM 113200007.

The second author was supported by NSF Grant DMS-0097881.

(C)2004 American Mathematical Society Reverts to public domain 28 years from publication 
not in Stegall's class, and $\left(Y^{*}, w^{*}\right)$ is in Stegall's class but is not fragmentable" is consistent as well.

Measurable cardinals are discussed in detail in [8]. They are commonly used in set-theoretic arguments, although their consistency strength is known to be stronger than just ZFC; that is, their relative consistency (i.e., the implication $\mathrm{Con}(\mathrm{ZFC}) \Rightarrow$ $\mathrm{Con}(\mathrm{ZFC}+$ there is a measurable cardinal)) cannot be proved in $\mathrm{ZFC}$, and there is no procedure for constructing a model of "ZFC + there is a measurable cardinal" from a model of ZFC. For a detailed explanation see [8].

We do not know if the measurable cardinal can be removed from the hypothesis of the Theorem, although it is needed for some of the lemmas. For example, the situation obtained in Lemma 6 implies the existence of an inner model with a measurable cardinal.

To prove the Theorem, we formulate the following set of axioms:

Axioms $\mathcal{A}$. (i) Martin's Axiom and $2^{\aleph_{0}}=\aleph_{3}$ hold.

(ii) There is a precipitous ideal over $\omega_{2}$.

(iii) The cardinal $\aleph_{1}$ is not measurable in any transitive model of ZFC containing all the ordinals.

We first prove (Lemma 2) the consistency of $\mathcal{A}$, and then construct $X$ and $Y$ using $\mathcal{A}$. In proving Lemma 2 , we use forcing to obtain (i) and (ii), but first we observe that (iii) cannot be forced to be false if it is true in the ground model.

Definition. For an ordinal $\rho$ let $\Psi(\rho)$ denote the formula " $\rho$ is a measurable cardinal in some transitive model of ZFC containing all the ordinals".

Lemma 1. Let $\rho$ be an ordinal such that $\neg \Psi(\rho)$ holds, and let $P$ be a partially ordered set. Then $1 \Vdash_{P} \neg \Psi(\rho)$.

Proof. First, define a $\rho$-model to be a transitive $M$ containing all the ordinals such that $M$ satisfies ZFC plus the statement $\rho$ is measurable and the universe (i.e., $M$ ) equals $L[u]$, where $u$ is a normal ultrafilter on $\rho$. By [10, this $M$ is unique (if it exists), so call it $M_{\rho}$. Furthermore, by [14], $M_{\rho} \models \mathrm{GCH}$, and, by [10], in $M_{\rho}$, the normal ultrafilter $u$ is unique. Now, $u$ is a subset of the power set $\mathcal{P}(\rho)$, but $u$ can be uniquely coded by a set of ordinals. Let $\Phi(\rho, \delta, W)$ assert that $\Psi(\rho)$ holds, $\delta=\left(\rho^{+}\right)^{M_{\rho}}$, and $W \subset \delta$ is the first subset of $\delta$ which codes the normal ultrafilter. So, $\Psi(\rho) \Rightarrow \exists ! \delta, W \Phi(\rho, \delta, W)$. Here, "first" is in the canonical order of construction from $u$, and "codes" can be via any standard method; for example, $u$ is the collection of all sets of the form $\{\xi<\rho: \rho \cdot \alpha+\xi \in W\}$, for $\alpha<\delta$.

Now, if 1 does not force $\neg \Psi(\rho)$, we may fix a $p \in P$, an ordinal $\delta$, and a name $\dot{W}$ such that $p \Vdash \Phi(\rho, \delta, \dot{W})$. Replacing $P$ with $\{q: q \leq p\}$, we can in fact assume that $1 \Vdash \Phi(\rho, \delta, \dot{W})$. Now, let $Q=P_{1} \times P_{2}$, where $P_{1}, P_{2}$ are isomorphic copies of $P$, and let $\dot{W}_{i}$ be the $P_{i}$-name corresponding to the $P$-name $\dot{W}$, so that $1 \Vdash_{Q} \Phi\left(\rho, \delta, \dot{W}_{1}\right) \wedge \Phi\left(\rho, \delta, \dot{W}_{2}\right)$, and hence, by uniqueness $1 \Vdash_{Q} \dot{W}_{1}=\dot{W}_{2}$. But then, since the names are disjointly supported, there is a $W \subseteq \delta$ in the ground model such that $1 \Vdash_{Q} \dot{W}_{1}=\dot{W}_{2}=W$, so that $\Psi(\rho)$ holds in the ground model.

Lemma 2. If "ZFC + there exists a measurable cardinal" is consistent, then " $Z F C$ $+\mathcal{A} "$ is consistent.

Proof. By [14], we may assume that the ground model $V$ satisfies $V=L[u]$ where $u$ is a normal ultrafilter on a measurable cardinal $\kappa$. Then, by [9, Theorem 9.4] we have $\neg \Psi\left(\omega_{1}\right)$ in $V$. 
Now consider the iterated generic extension $V\left[G_{1}\right]\left[G_{2}\right]$, where $G_{1}$ is the Levy collapse with countable conditions, making $\kappa=\omega_{2}$, and then $G_{2}$ is the ccc extension which makes Martin's Axiom and $2^{\aleph_{0}}=\aleph_{3}$ hold (see [15] or [11]). Since both these extensions preserve $\omega_{1}$, the validity of (iii) in $V\left[G_{1}\right]\left[G_{2}\right]$ follows from Lemma 1 . The validity of (i) is obvious. Furthermore, it follows from [3] that $\kappa$ carries a normal precipitous ideal in $V\left[G_{1}\right]$, and this is true also in $V\left[G_{1}\right]\left[G_{2}\right]$, since such ideals are preserved by ccc extensions by 4 .

Now we give a series of lemmas which prove the Theorem from $\mathcal{A}$.

Lemma 3 (Under $\mathcal{A}$ ). Let $X$ be a Baire space and $\mathcal{E}$ a disjoint family of meager subsets of $X$ such that $\operatorname{card} \mathcal{E}=\aleph_{1}$ and $\bigcup \mathcal{E}^{\prime}$ has the Baire property in $X$ for every $\mathcal{E}^{\prime} \subset \mathcal{E}$. Then $\bigcup \mathcal{E}$ is meager in $X$.

Proof. Suppose $\bigcup \mathcal{E}$ is not meager. Then we can suppose without loss of generality that $X=\bigcup \mathcal{E}$. Now, by the Lemma in the proof of Theorem 3.3 in [2], card $\mathcal{E}=\aleph_{1}$ is a measurable cardinal in some submodel, contradicting (iii).

The following lemma is a special case of [5, Proposition 2.4].

Lemma 4. Let $X$ be a Baire space of weight at most $\kappa$ (where $\kappa$ is an uncountable cardinal). Let $\mathcal{E}$ be a disjoint family of meager sets such that $\bigcup \mathcal{E}^{\prime}$ is $F_{\sigma}$ in $X$ for all $\mathcal{E}^{\prime} \subseteq \mathcal{E}$, and such that $\bigcup \mathcal{E}^{\prime}$ is meager in $X$ for all $\mathcal{E}^{\prime} \subseteq \mathcal{E}$ with card $\mathcal{E}^{\prime}<\kappa$. Then $\bigcup \mathcal{E}$ is meager in $X$.

Proof. Suppose that $\bigcup \mathcal{E}$ is not meager. Since $\bigcup \mathcal{E}$ is $F_{\sigma}$, its interior $G=\operatorname{int} \bigcup \mathcal{E}$ is nonempty. Let $\left\{U_{\alpha}: \alpha<\kappa\right\}$ be an open base for $G$. Choose by transfinite induction sets $E_{\xi}, F_{\xi} \in \mathcal{E}$ for $\xi<\kappa$ such that

(a) $E_{\xi} \cap U_{\xi} \neq \emptyset$ and $F_{\xi} \cap U_{\xi} \neq \emptyset$;

(b) $E_{\xi}, F_{\xi} \notin\left\{E_{\eta}, F_{\eta}: \eta<\xi\right\}$;

(c) $F_{\xi} \neq E_{\xi}$.

This is possible because each $\bigcup\left\{E_{\eta} \cup F_{\eta}: \eta<\xi\right\}$ is meager. Let $H_{1}=\bigcup\left\{E_{\xi} \cap G\right.$ : $\xi<\kappa\}$ and $H_{2}=G \backslash H_{1}$. Then both $H_{1}$ and $H_{2}$ are dense $F_{\sigma}$ in $G$, so both are meager; hence $G$ is meager, a contradiction.

By Lemmas 3 and 4 we get:

Lemma 5 (Under $\mathcal{A})$. Let $X$ be a Baire space of weight at most $\aleph_{2}$ and $\mathcal{E}$ be a disjoint family of meager sets such that $\bigcup \mathcal{E}^{\prime}$ is $F_{\sigma}$ for any $\mathcal{E}^{\prime} \subseteq \mathcal{E}$. Then $\bigcup \mathcal{E}$ is meager in $X$.

We continue by the following consequence of [2].

Lemma 6 (Under $\mathcal{A})$. There is a Baire metric space $X$ of weight $\aleph_{3}$ and a decomposition $\mathcal{E}$ of $X$ into meager sets such that $\bigcup \mathcal{E}^{\prime}$ has the Baire property in $X$ for any $\mathcal{E}^{\prime} \subset \mathcal{E}$ and $\operatorname{card} \mathcal{E}=\aleph_{2}$.

Proof. In [2, Theorem 3.2] there is constructed, under (ii), such a decomposition of a Baire metric space of weight $2^{\aleph_{2}}$, which is $\aleph_{3}$ by (i) (see [13]).

Now we are almost ready to prove the Theorem. It remains to recall the definition of Stegall spaces with respect to a class of Baire spaces, the construction from [6] and a lemma from [7].

Let $\mathcal{C}$ be a class of Baire spaces closed under taking open subsets and dense Baire subspaces. We say that a topological space $T$ is Stegall with respect to $\mathcal{C}$ if for any 
$B \in \mathcal{C}$ and any upper-semicontinuous compact-valued mapping $\varphi: B \rightarrow T$, which is minimal with respect to inclusion, $\varphi(b)$ is a singleton for all $b \in B$ except for a meager set.

If $A \subset(0,1)$ is arbitrary, we put $K_{A}=((0,1] \times\{0\}) \cup((\{0\} \cup A) \times\{1\})$ and equip this set with the lexicographical order (i.e., $(s, a)<(t, b)$ if either $s<t$, or $s=t$ and $a<b$ ) and the order topology. By [6] Proposition 2] the space $K_{A}$ is compact and Hausdorff.

Lemma 7. Let $\mathcal{C}$ be a class of Baire spaces which is closed under taking open subspaces and dense Baire subspaces, and let $A \subset(0,1)$. Consider:

(1) $\left(C\left(K_{A}\right)^{*}, w^{*}\right)$ is Stegall with respect to $\mathcal{C}$.

(2) For any $B \in \mathcal{C}$ and any continuous $f: B \rightarrow A$, there is a nonempty open $U \subset B$ such that $f$ is constant on $U$.

Then $(2) \Rightarrow(1)$; and $(1) \Rightarrow(2)$ when all the spaces in $\mathcal{C}$ are metrizable.

$(2) \Rightarrow(1)$ is proved in $[9]$, and $(1) \Rightarrow(2)$ is proved in [7].

Now the Theorem is an immediate consequence of Lemma 2 and the following lemma.

Lemma 8 (Under $\mathcal{A}$ ). Let $A \subset(0,1)$ be arbitrary.

(a) $\left(C\left(K_{A}\right)^{*}, w^{*}\right)$ is fragmentable if and only if $A$ is countable.

(b) $\left(C\left(K_{A}\right)^{*}, w^{*}\right)$ is Stegall if and only if card $A \leq \aleph_{1}$.

(c) If card $A \leq \aleph_{2}$, then $C\left(K_{A}\right)$ is weak Asplund.

Proof. (a) is proved in [6, Proposition 3] and requires no special axioms of set theory.

$(\mathrm{b}) \Leftarrow$ : It is enough to show that $A$ satisfies condition (2) of Lemma 7 with respect to the class of all Baire spaces. Let $B$ be a Baire space and $f: B \rightarrow A$ continuous. The family $\left\{f^{-1}(a): a \in A\right\}$ is a partition of $B$. Moreover, the union of each subfamily is $F_{\sigma}$, since each subset of $A$ is relatively $F_{\sigma}$ by Martin's Axiom (see [12, p.162]). Hence, by Lemma 3, some $f^{-1}(a)$ is non-meager and therefore has a nonempty interior.

(b) $\Rightarrow$ : Let card $A \geq \aleph_{2}$. Let $X$ be the metric space with partition $\mathcal{E}$ from Lemma 6. Let $\phi: \mathcal{E} \rightarrow A$ be a one-to-one mapping. Define $\tilde{f}: X \rightarrow A$ by the formula $\tilde{f}(x)=a$ if $a=\phi(E)$ such that $x \in E$. Then $\tilde{f}$, considered as a function to $\mathbb{R}$, has the Baire property and so there is (by [12]) a residual $B \subset X$ such that $f=\tilde{f} \uparrow B$ is continuous. Hence $A$ does not satisfy condition (2) of Lemma 7.

(c): It is enough to show that $\left(C\left(K_{A}\right)^{*}, w^{*}\right)$ is Stegall with respect to Baire metric spaces of weight at most $\aleph_{2}$. Since each subset of $A$ is again relatively $F_{\sigma}$, the proof is the same as in $(\mathrm{b}) \Leftarrow$, only use Lemma 5 instead of Lemma 2 .

Let us finish by mentioning the following open question.

Problem. Is it consistent with ZFC to suppose that any weak Asplund space has weak $^{*}$ fragmentable dual?

\section{REFERENCES}

1. M. Fabian, Gâteaux differentiability of convex functions and topology: weak Asplund spaces, Wiley-Interscience, New York, 1997. MR 1461271 (98h:46009)

2. R. Frankiewicz and K. Kunen, Solution of Kuratowski's problem on function having the Baire property I., Fund. Math. 128 (1987), 171-180. MR 0922569 (89a:03090) 
3. T. Jech, M. Magidor, W. Mitchell and K. Prikry, Precipitous ideals, J. Symb. Log. 45 (1980), 1-8. MR 0560220 (81h:03097)

4. Y. Kakuda, On a condition for Cohen extensions which preserve precipitous ideals, J. Symbolic Logic 46 (2) (1981), 296-300. MR 0613283 (82i:03062)

5. O. Kalenda, Hereditarily Baire spaces and point of continuity property, Diploma Thesis, Charles University, Prague, 1995 (Czech).

6. O. Kalenda, Stegall compact spaces which are not fragmentable, Topol. Appl. 96 (2) (1999), 121-132. MR 1702306 (2000i:54027)

7. O. Kalenda, A weak Asplund space whose dual is not in Stegall's class, Proc. Amer. Math. Soc. 130 (7) (2002), 2139-2143. MR 1896051 (2003a:46024)

8. A. Kanamori, The higher infinite. Large cardinals in set theory from their beginnings, Springer-Verlag, Berlin, 1994. MR 1321144 (96k:03125)

9. P. Kenderov, W. Moors and S. Sciffer, A weak Asplund space whose dual is not weak* fragmentable, Proc. Amer. Math. Soc. 129 (12) (2001), 3741-3747. MR 1860511 (2002h:54014)

10. K. Kunen, Some applications of iterated ultrapowers in set theory, Ann. Math. Logic 1 (1970), 179-227. MR 0277346 (43:3080)

11. K. Kunen, Set theory. An introduction to independence proofs, Studies in logic and the foundations of mathematics, vol. 102, North-Holland, 1980. MR 0597342 (82f:03001)

12. C. Kuratowski, La propriété de Baire dans les espaces métriques, Fund. Math. 16 (1930), 390-394.

13. D. A. Martin and R. M. Solovay, Internal Cohen extensions, Ann. Math. Logic 2 (1970), 143-178. MR 0270904 (42:5787)

14. J. Silver, The consistency of the GCH with the existence of a measurable cardinal, Axiomatic Set Theory, Proc. Sympos. Pure Math., Vol. XIII, Part I,, Univ. California, Los Angeles, Calif., 1967, pp. 391-395. MR 0278937 (43:4663)

15. R. M. Solovay and S. Tennenbaum, Iterated Cohen extensions and Souslin's problem, Ann. of Math. 94 (1971), 201-245. MR 0294139 (45:3212)

16. C. Stegall, A class of topological spaces and differentiability, Vorlesungen aus dem Fachbereich Mathematik der Universität Essen 10 (1983), 63-77. MR 0730947 (85j:46026)

Department of Mathematical Analysis, Charles University, Sokolovská 83, 18675 Praha 8, Czech Republic

E-mail address: kalenda@karlin.mff.cuni.cz

Department of Mathematics, University of Wisconsin, Madison, Wisconsin 53706

E-mail address: kunen@math.wisc.edu 